\title{
UNE ÉTUDE COMPARATIVE DE LA LANGUE DES SIGNES QUÉBÉCOISE (LSQ) ET LA LANGUE BRÉSILIENNE DES SIGNES (LIBRAS): QUELQUES ASPECTS LINGUISTIQUES, SOCIO-POLITIQUE-CULTURELS ET HISTORIQUES
}

UM ESTUDO COMPARATIVO ENTRE A LÍNGUA DE SINAIS QUEBEQUIANA (LSQ) E A LINGUA BRASILEIRA DE SINAIS (LIBRAS): ALGUNS ASPECTOS LINGUÍSTICOS, SOCIO-POLÍTICOS-CULTURAIS E HISTÓRICOS

\section{A COMPARATIVE STUDY BETWEEN THE QUEBECIAN SIGN LANGUAGE (LSQ) AND THE BRAZILIAN LANGUAGE OF SIGNS (LIBRAS): SOME LANGUAGE, SOCIO-POLITICAL, CULTURAL AND HISTORICAL ASPECTS}

\author{
Luiz Maurício Rios * \\ Valdirene Maria de Araújo Gomes **
}

\section{Résumé}

L'objectif principal de cet article est de promouvoir la connaissance et la comphéhension des Études Sourdes au Canada et au Brésil, à partir d'études comparatives de la Langue des Signes Québécoise (LSQ) et la Langue Brésilienne des Signes (LIBRAS). Nous faisons, aussi, une brève approche de quelques aspects linguistiques, socio-politique-culturels et historiques de la Culture Sourde, avec l'aspiration de démontrer quelques études liées aux questions de l'enseignement des langues des signes et à l'inclusion de l'individu sourd à la société, en spécial, l'histoire de l'origine de l'enseignement et des recherches dans ce domaine. Il est pertinent de signaler que cet article s'inscrit dans le cadre des études comparées, ce qui pourra être une ouverture au dialogue entre les différentes recherches et approches sur ce thème, développées dans ces deux pays américains, afin de chercher ensemble des solutions stratégiques d'inclusion sociale et d'élaboration de politiques de diversité culturelle : la culture orale et la culture des sourds.

Mots-Clés : Langue des Signes Québécoise (LSQ). Langue Brésilienne de Signes (LI BRAS). Bilinguisme. Enseignement des langues des signes.

\footnotetext{
* Mestre em Letras e Linguística, professor na Universidade Federal de Goiás, na Área de Francês. E-mail: 1mrios42@gmail.com

** Mestre em Letras e Linguística, professora na Universidade Federal de Goiás, na Área de Inglês. E-mail: val.letrasufg@gmail.com
}

Revista Sinalizar, Goiânia, v. 2, n.2, p. 235 - 245, jul. / dez., 2017. 
Dans le silence, il y a un univers où la communication est possible à partir de signes. La langue des signes est la langue utilisée principalement par les sourds. Cette langue partage plusieurs caractéristiques avec les langues orales, par exemple une grammaire, une syntaxe, une pragmatique, un lexique, une classification etc. Cette langue visuelle est le moyen de communication utilisé par les sourds pour pouvoir interagir avec la réalité extérieure. Elle est une langue à part entière au même titre que les langues parlées, telles que le Français ou l'Anglais.

Largement utilisée chez les sourds, la langue des signes n'est pas la même d'un pays à l'autre. Chaque pays a sa propre Langue des Signes que les sourds utilisent. Elle n'est pas universelle, mais les sourds de pays différents se communiquent facilement entre eux après une courte période d'adaptation. À titre d'exemple, citons la L.S.F. (Langue des Signes Française), la B.S.L. (British Sign Language), la A.S.L.( American Sign Language) et la L.S.Q. (Langue des Signes Québécoise). Au Brésil c'est Libras (Língua Brasileira de Sinais). Et c'est ainsi par d'autres pays.

La langue des signes n'est pas une langue universelle. Le Summer Institute of Linguistics (SIL International) recense 130 langues des signes différentes dont 126 actuellement utilisées comme première langue par environ 25,5 millions de personnes à travers le monde. Il existe en fait, tout comme pour le langage oral, autant de langues des signes que de communautés différentes de sourds. Chaque langue des signes ayant son histoire, ses unités signifiantes et son lexique.

Le développement d'une langue des signes dépend de la vivacité de la communauté des personnes qui la composent, comme pour une langue vocale. La langue des signes d'une région n'a pas nécessairement une correspondance avec la langue orale régionale. En dépit des différences entre les langues des signes du monde, la compréhension et la communication est rapidement possible entre deux personnes maîtrisant des langues des signes différentes. Ceci tient à la grande proximité des structures syntaxiques et à l'existence de structures très iconiques, caractérisées par l'absence de signes standard (qui sont eux différents pour chaque langue). L'origine de ces structures partagées tient

Revista Sinalizar, Goiânia, v. 2, n.2, p. 235 - 245, jul. / dez., 2017. 
probablement à la nature même de la langue et transfigure l'expérience humaine du monde qu'en ont ses locuteurs (BERTIN, 2010).

Il faut, alors et avant tout, comprendre qu'un sourd est sourd, et un muet est muet. Les sourds ne sont pas muets et les muets ne sont pas sourds. En fait, très peu d'entre-eux se classent dans la catégorie "Sourds-muets". Le "politiquement correcte" fait qu'on distingue maintenant les sourds des malentendants, alors qu'il s'agit de deux groupes différents. Un malentendant pourrait entendre avec un appareil, alors qu'un sourd profond ne pourra jamais entendre. Enfin, il ne faut pas dire "parler" la langue des signes, mais "communiquer" par la langue des signes.

La langue des signes est actuellement pratiquée par des personnes sourdes et les professionnels en contact avec eux, comme les enseignants, orthophonistes, travailleurs sociaux et les interprètes spécialisés. Elle peut également être utile aux devenus-sourds, aux travailleurs dans l'industrie bruyante, aux plongeurs sous-marins et dans tout autre contexte où la communication verbale n'est pas possible. Elle est constituée de 5 paramètres: positions des doigts et de la main, mouvements, emplacement et expressions du visage. Elle comprend également une syntaxe (le lieu, les personnages, l'action) et une grammaire (MACHABÉE, 1998).

La culture peut être définie comme l'ensemble des détails du quotidien, des traditions qui constituent le mode de vie typique à un groupe social. La culture sourde est extrêmement riche. Les sourds vivent dans un monde visuel, leur mode d'expression est structuré par la vision. On peut donner comme exemple de différence culturelle le fait que dans le monde des sourds, il est fréquent de se toucher, se taper sur l'épaule, gestes qui sont moins bien adoptés par les entendants.

Aujourd'hui encore, faute d'information, de nombreuses personnes sourdes ou parents de sourds ne connaissent pas l'existence des langues des signes et considèrent avant tout la surdité comme un handicap. Il semble nécessaire d'avoir une approche différente de la simple vision curative de la surdité et de prendre en considération la réalité sociale et linguistique des langues des signes. De nombreux pays souhaitent avant tout un épanouissement des personnes et développent l'accès en langue des signes aux lieux

Revista Sinalizar, Goiânia, v. 2, n.2, p. 235 - 245, jul. / dez., 2017. 
publics, aux universités etc.

Seules quelques-unes, de la centaine de langues des signes dans le monde, ont obtenu une reconnaissance légale, les autres ne bénéficiant d'aucun statut officiel. Certains pays ont signés des lois pour la reconnaissance de la langue, la culture et la citoyenneté des sourds. En France, la loi n² 2005-102 du 11 février 2005, pour l'égalité des droits et des chances, la participation et la citoyenneté des personnes handicapées, reconnaît officiellement la LSF. Au Canada, la province du Manitoba est la première à reconnaître officiellement la langue des signes américaine comme celle des communautés sourdes en milieu anglophone, en 1988, suivi de l'Alberta qui reconnaît l'ASL comme langue optionnelle dans l'enseignement, en 1990; l'Ontario reconnaît l'ASL et la LSQ comme langues d'enseignement, en 1993. Aux États-Unis, la American Sign Language est reconnue dans plusieurs états en tant que langue étrangère. Au Brésil, la Langue des Signes Brésilienne (LIBRAS) est reconnue officiellement en 2002 dans le domaine de l'éducation. Il est statué que chaque enfant sourd a le droit d'apprendre en sa propre langue et d'avoir le portugais comme deuxième langue (BRASIL, 2002).

En 2009, pour contribuer à la valorisation et à l'inclusion sociale des sourds, 1'Universidade Federal de Goiás, au centre du Brésil, inaugure le premier Cours Universitaire de Lettres - Libras présentiel, au Brésil. Il s'agit d'une Licence à laquelle s'inscrivent 40 étudiants par an. À l'année 2011, il y a eu 25 places pour des sourds. Pour les sourds brésiliens, la langue maternelle n'est pas la langue portugaise, mais Libras. La Loi 10.436, de 2002, et le Décret 5626, de 2005, déterminent que les Universités créent le Cours de Lettres - Libras. Pour les autres Cours de Licence, comme Mathématiques et Histoire, la discipline de Libras est obligatoire (BRASIL, 2002; 2005).

Il est important de signaler que les études des Langues des Signes Canadiennes ne sont pas très bien connues du public Brésilien et nous sommes convaincus que la réalisation de cet article engendrera des réflexions et des discussions menant à de nouvelles approches concernant le traitement des questions éducationnelles, politiques et pratiques qui touchent les citoyens canadiens et brésiliens qui affrontent, quotidiennement, le défis de vivre dans des sociétés qui ne sont pas encore entièrement organisées pour pouvoir offrir à la

Revista Sinalizar, Goiânia, v. 2, n.2, p. 235 - 245, jul. / dez., 2017. 
population sourde l'égalité d'opportunités, mais qui parcourent toutes les deux, la canadienne et la brésilienne, des voies qui visent à offrir à ce groupe, considéré minoritaire, des conditions réelles d'inclusion sociale et d'accès au plein droit d'exercer la démocratie.

Il est pertinent de signaler que j’ai eu l'opportunité de présenter le sujet de ces études à partir de la discipline intitulée "Estudo Comparativo entre LIBRAS e LSQ : aspectos linguísticos e sócio-político-culturais", présentée aux étudiants du Cours de Lettres, du Cours de Lettres - LIBRAS et à d'autres Cours de mon Université, aussi bien qu'aux membres de la communauté sourde de notre région et aux intéressés aux études des langues des signes, bien que pour les études canadiennes. L'opportunité de présenter la LSQ, en faisant une comparaison avec la LIBRAS, c'est un aspect qui pourra enrichir l'apprentissage de ces étudiants. Pour parler de la recherche qui se fait sur la langue des signes québécoise à Montréal, nous commencerons avec une présentation démographique et socio-politique de la LSQ au Canada.

Les langues des signes au Canada sont au nombre de cinq. Les deux principales sont la LSA et la LSQ. En fait au Canada on dénombre un nombre de Sourds à 35470 personnes. Quand on dit Sourd, Sourd avec un grand S, ce sont les Sourds culturellement sourds, qui s'identifient à la culture sourde et qui utilisent unelangue des signes comme langue de référence au Québec, qui est une des provinces canadiennes qui comprend le plus grand nombre de personnes sourdes, c'est la deuxième après l'Ontario. Au Québec, on compte environ 6500 Sourds, dont la langue des signes est la langue de référence (BOUCHARD, 2004). En ce qui concerne les démarches de reconnaissance de la LSQ, ce qui est bon de savoir en partant c'est que la LSQ n'est pas reconnu, ni comme langue d'enseignement, ni comme langue officielle.

Au Canada, il existe deux langues officielles, ce sont le français et l'anglais. Si on parle de langue d'enseignement, ce qui est bon de connaître c'est que l'enseignement relève d'un pouvoir provincial donc ce n'est pas au niveau fédéral, au niveau canadien que se décide les actions liées à l'enseignement, mais chaque province décide des actions liées à l'enseignement. Au Québec, la seule langue officielle d'enseignement c'est le français.

Revista Sinalizar, Goiânia, v. 2, n.2, p. 235 - 245, jul. / dez., 2017. 
Actuellement ce fait politique est dû à la situation particulière du français en Amérique du Nord et la situation particulière du français au Canada. Par souci de protection de la langue il y a la loi 101, votée dans les années 1977, qui protège finalement l'éducation en fait qui empêche l'éducation de se faire dans une autre langue que le français donc sauf quelques rares exceptions toutes les écoles francophones, toutes les écoles au Québec utilisent le français comme langue d'enseignement. C'est entr'autres une embûche pour le développement pour le statut de la LSQ comme langue d'enseignement (TERMOTE, 1988).

Les revendications des Sourds québécois pour la reconnaissance de la LSQ comme langue officielle est de toujours, mais elle a particulièrement pris de l'ampleur et du corps dans les années '80. Les Sourds se sont regroupés pour créer une association officielle des enseignants de la LSQ pour trouver des moyens, pour officialiser les moyens, les méthodes d'enseignement de la langue des signes mais il y a aussi les méthodes de formation des professeurs sourds qui enseignaient la LSQ. Ce premier pas, cette première action a été le début d'une série d'actions qui se sont enchaînées pour officialiser les démarches politiques dans le but d'obtenir du MEQ (Ministère de l'Éducation du Québec) qu'il y ait une reconnaissance officielle de la LSQ.

Dans les annés '90 il y a eu une demande de reconnaissance officielle de la LSQ comme langue d'enseignement par un regroupement d'association sourde où on a déposé un mémoire au MEQ pour faire la première demande officielle. À cette époque, il y a eu une action, pas liée à cette première action, qui a été une demande officielle de la part de l'association des parents entendants d'enfants sourds pour que le MEQ fasse finalement une expérimentation de l'approche bilingue LSQ/français dans une école spécialisée de Montréal. Donc les parents, compte tenu des actions politiques par les sourds, compte tenu de ce qu'on savait des bienfaits de l'approche bilingue par les recherches américaines, ont demandé au MEQ d'expérimenter l'approche (PARISOT, 2002).

Dans les années 2000, il y a eu un événement politique qui était tout à fait en dehors des actions de la communauté sourde mais qui a été récupéré par la communauté sourde pour promouvoir, pour pousser et pour réitérer la demande de reconnaissance de LSQ. Cet

Revista Sinalizar, Goiânia, v. 2, n.2, p. 235 - 245, jul. / dez., 2017. 
événement était les États généraux sur la situation et l'avenir de la langue française au Québec. Donc le ministère de la culture avait mandaté une commission pour aller rencontrer différents intervenants dans toutes les régions de la province pour connaître leur point de vue sur la situation de la langue française au Québec dans l'enseignement mais aussi dans la vie quotidienne.

Les Sourds de la communauté ont profité de l'occasion pour déposer un mémoire lors de cet événement revendiquant principalement la reconnaissance de la LSQ. Donc le regroupement des organismes sourds comptait, entr'autres, des organismes provinciaux, un organisme qui a pour mandat la protection de la langue et de la culture des sourds du Québec; le regroupement des enseignants de LSQ; et aussi des alliés entendants concernés par la langue et l'enseignement en surdité dont l'association des parents, des centres de réadaptation, des écoles, des commissions scolaires etc. Donc il y avait un regroupement de personnes importantes qui ont signé un mémoire qui a été remis à la commission des états généraux de la langue, qui présentait une série d'arguments pour la reconnaissance de la LSQ.

Ces arguments ont été mis de l'avant dans le rapport qui a été déposé à la commission. La démarche de ces organismes a été accueilli favorablement et il y a eu une recommandation de reconnaissance la LSQ comme langue première des sourds et que le MEQ la reconnaisse comme langue d'enseignement dans une relation complémentaire de bilinguisme LSQ/français. Il y avait recommandation que l'article 72, un article de la charte de la langue française québécoise, soit modifié en conséquence. Non seulement que soit reconnu par le gouvernement mais que soit inscrit dans la Charte. Aussi la commission a souligné qu'une société ne peut pas se permettre que des catégories de citoyens soient ignorés dans la reconnaissance de leur droit. Cela été un événement important parce que c'est inscrit, dans le texte du rapport de la commission, que la LSQ participe à l'intégration des sourds comme citoyen dans la société et fasse partie d'un tout. Malheureusement, malgré cette recommandation qui a été déposée au ministère d'état à la culture, la LSQ n'a pas été reconnu comme langue officielle d'enseignement.

Revista Sinalizar, Goiânia, v. 2, n.2, p. 235 - 245, jul. / dez., 2017. 
Alors, on disait qu'une reconnaissance officielle de la LSQ signifierait qu'il y aurait obligation d'offrir l'enseignement dans cette langue. Les enjeux d'une telle reconnaissance sont humains, financiers et organisationnels n'étaient pas décrits comme supportable par la direction de l'adaptation scolaire.Suite à cet échec, la communauté sourde a demandé á l'OPHQ (Office des personnes handicapées du Québec) de faire un rapport sur l'état de la LSQ. De ce rapport sont nés des groupes de travail, donc l'OPHQ, en concertation avec le MEQ qui ont mis sur pied deux groupes de travail à la suite des recommandations du rapport. Jusqu'à ce jour, il n’y a aucune action du MEQ qui a été posée en faveur de la reconnaissance de la LSQ comme langue d'enseignement.

Les conclusions de l'expérimentation de l'approche bilingue ont montré des résultats positifs sur les plans tant académique que sur le développement culturel et social des élèves. L'expérimentation a duré pendant 6 ans et, àchaque année, il y a eu des rapports préliminaires de recherche qui montrent une évolution dans la formation du personnel, l'évolution dans le développement du matériel, l'évolution dans l'évaluation des compétences linguistiques des enfants et le rapport final montre qu'effectivement les bienfaits d'une approche qui privilégie le passage des acquis d'une langue des signes vers des acquis linguistiques dans la forme de la langue majoritaire en ce qui nous concerne le français oral (VERCAINGNE-MENARD et al, 2005).

Les recommandations des chercheurs suite à cette expérimentation, sont pour l'implantation efficiente de l'approche. Si le MEQ souhaitait implanter l'approche pour que ça se fasse de façon efficiente, durable et que l'implantation ait des chances de succès, devait passer par la reconnaissance de la langue d'enseignement, entr'autres ne serait-ce que pour tant les professeurs que les enfants aient une image positive de leur apprentissage et de leur enseignement.

La situation actuelle c'est que malgré les résultats et les recommandations dela recherche, malgré le constat évoqué par le MEQ en 2003 visant à justifier la non reconnaissance de la LSQ comme langue d'enseignement, il n'existe toujours pas en 2010 de reconnaissance officielle ni de la langue ni de l'approche. Les maîtres ne sont toujours pas formés et la formation des interprètes n'est toujours pas reconnu. L'approche bilingue

Revista Sinalizar, Goiânia, v. 2, n.2, p. 235 - 245, jul. / dez., 2017. 
elle est née au Québec et aussi dans les autres pays qui utilisent l'approche bilingue en surdité. Elle est née de l'échec des méthodes traditionelles. Au Québec plusieurs avaient préalablement démontré que la communication totale pour les enfants gestuels menaient à des échecs tant dans l'apprentissage des contenus linguistiques que dans l'épanouissement de soi, le sentiment d'appartenance, tant dans les aspects sociaux et psychologiques du développement.

Cette conscience pourra être mise à profit et on pousse l'enfant à le mettre à profit dans l'apprentissage du français écrit. Il y a de constantes aller-retour tout en séparant toujours et l'apprentissage des notions grammaticales dans les deux langues. L'approche bilingue est bilingue mais aussi parce que l'enfant est sensibilisé au fait que les deux langues appartiennent à deux cultures différentes. Il navigue entre deux cultures. Lui-même est porteur de ces deux langues et de ces deux cultures (PARISOT, 2002).

Il y a un enseignement de la langue des signes pas seulement en langue des signes mais de la langue des signes que les élèves reçoivent, des contenus scolaires et des exercices sur la grammaire de la LSQ. Et il y a un enseignement de la langue des signes c'est-à-dire qu'en classe les contenus sont transférés en français et en LSQ. L'approche bilingue en surdité telle a été implantée sur la base des 6-8 années d'expérimentation, selon lesquelles il y a une corrélation entre la maîtrise de la LSQ et la maîtrise en lecture. Un enfant qui maîtrise bien la LSQ va avoir une bonne maîtrise globale de la lecture et inversement un enfant qui maîtrise mal la LSQ va avoir une moins bonne maîtrise globale du français.

La discussion des problématiques qui entourent la culture et l'univers des sourds pourra dégager des réflexions salutaires qui produiront, certainement, des propositions d'élaboration des politiques d'inclusion sociale tout en attirant l'attention vers l'urgence du respect à la diversité culturelle. Pour conclure, nous attendons avoir promu la connaissance et la compréhension du Canada au Brésil, en ouvrant un dialogue et des questions sur les aspects linguistiques, socio-politique-culturels et historiquesdans ce thème, avec quelques questions de l'enseignement des langues des signes et de l’inclusion de l'individu sourd à la société.

Revista Sinalizar, Goiânia, v. 2, n.2, p. 235 - 245, jul. / dez., 2017. 


\title{
Resumo
}

O objetivo principal deste artigo é promover o conhecimento e a compreensão dos Estudos Surdos no Canadá e no Brasil, a partir de estudos comparativos entre a Língua de Sinais Quebequense (LSQ) e a Língua Brasileira de Sinais (LIBRAS). Também se faz uma breve abordagem de alguns aspectos linguísticos, sócio-político-culturais e históricos da Cultura Surda, objetivando apresentar alguns estudos ligados às questões do ensino das línguas de sinais e à inclusão do indivíduo surdo na sociedade, focando, especialmente, a história da origem do ensino e das pesquisas nesse domínio. É importante assinalar que este artigo se insere no quadro dos estudos comparados, o que poderá ser uma abertura para o diálogo entre as diferentes pesquisas e abordagens sobre o tema, desenvolvidos nesses dois países americanos, a fim de procurar, juntos, soluções estratégicas de inclusão social e de elaboração de políticas de diversidade cultural: a cultura oral e a cultura surda.

Palavras-chave: Língua de Sinais Quebequense (LSQ). Língua Brasileira de Sinais (LIBRAS). Bilinguismo. Ensino de Língua de Sinais

\begin{abstract}
The main objective of this article is to promote the knowledge and understanding of Canada in Brazil, based on comparative studies of the Quebec Sign Language (QSL) and the Brazilian Sign Language (LIBRAS), and some linguistic, social, political and historical aspects, opening the dialogue on this subject. Another objective is to present some studies related to the issue of sign language teaching and the inclusion of deaf individuals in the society: the history of the origin of teaching and research in this field. It is relevant to point out that this article is part of the comparative studies, which may be the opening to the dialogue between different researches and approaches on this topic, developed in these two American countries, in order to seek, together, for strategic solutions of social inclusion and of cultural diversity policy making: oral culture and deaf culture.
\end{abstract}

Keywords: Quebec Sign Language (LSQ). Brazilian Sign Language (LIBRAS), Bilingualism. Sign Language Teaching.

\section{Références}

BERTIN, Fabrice. Ferdinand Berthier ou le rêve d'une nation sourde. M. Companys, 2010.

BRASIL. Decreto $n^{0}$ 5.626, de 22 de dezembro de 2005. Regulamenta a Lei $n^{\circ} 10.436$, de 24 de abril de 2002, que dispõe sobre a Língua Brasileira de Sinais - Libras, e o art. 18 da

Revista Sinalizar, Goiânia, v. 2, n.2, p. 235 - 245, jul. / dez., 2017. 
Lei no 10.098, de 19 de dezembro de 2000. Diário Oficial [da] União, Brasília, DF, 23 dez. 2005. Disponível em:

http://www.planalto.gov.br/ccivil_03/_ato20042006/2005/decreto/d5626.htm. Acesso em: 15 nov. 2017.

BRASIL. Lei $\mathbf{n}^{0}$ 10.436, de 24 de abril de 2002. Dispõe sobre a Língua Brasileira de Sinais - Libras e dá outras providências. Diário Oficial [da] União, Brasília, DF, 25 abr. 2002. Disponível em: <http://www.planalto.gov.br/ccivil_03/leis/2002/L10436.htm>. Acesso em: 15 nov. 2017.

FRANCE. Lei $\mathbf{n}^{\mathbf{0}}$ 2005-102, de 11 de fevereiro de 2005. Dispõe sobre a igualdade dos direitos e oportunidades, a participação e a cidadania de pessoas com deficiências. JORF, $\mathrm{n}^{\circ}$ 36 , de 12 de fevereiro de 2005, pág. 2353, texto $\mathrm{n}^{\circ} 1$. Disponível em <https://www.legifrance.gouv.fr/affichTexte.do?cidTexte=JORFTEXT000000809647\&cat egorieLien=id $>$. Acesso em: 15 nov.2017.

MACHABÉE, D. et A.-M. PARISOT. "Le point de vue des sourds", in Lecture, écriture et surdité: visions actuelles et nouvelles perspectives, Dubuisson et Daigle (éd.), Montréal: Les Éditions Logiques, p. 267-288, 1998.

TERMOTE, Marc et Danielle Gauvreau. La situation démolinguistique au Québec, Québec, Conseil de la langue française, 1988.

PARISOT, A.-M. L'option du bilinguisme LSQ-français en surdité : projet de diffusion des connaissances auprès des parents d'enfants sourds, conférence présentée à l'Assemblé générale de l'Association du Québec pour enfants avec problèmes auditifs (AQEPA), Montréal, 2002.

VERCAINGNE-MÉNARD, A., A.-M. PARISOT et C. DUBUISSON. L'approche bilingue à l'école Gadbois. Six années d'expérimentation. Bilan et recommandations, rapport déposé au ministère de l'Éducation du Québec. Montréal : Université du Québec à Montréal, 2005.

Revista Sinalizar, Goiânia, v. 2, n.2, p. 235 - 245, jul. / dez., 2017. 